\title{
Oferta diaria de pradera: Efecto sobre el rendimiento y calidad de la pradera y sobre la producción de leche por vaca y por hectárea
}

\author{
Daily herbage allowance: Effect on yield and quality of pasture \\ and on milk production per cow and per hectare
}

\author{
Balocchi, 0. ${ }^{a *}$, Pulido, R. ${ }^{b}$, Merino, V $^{a}$ \\ ${ }^{a}$ Instituto de Producción Animal, Facultad de Ciencias Agrarias, Universidad Austral de Chile. Valdivia, Chile. \\ ${ }^{b}$ Instituto de Ciencia Animal, Facultad de Ciencias Veterinarias, Universidad Austral de Chile. Valdivia, Chile.
}

A R T I C LE INF O

\begin{tabular}{l}
\hline Keywords: \\
Grazing management \\
Grazing efficiency \\
Stocking rate \\
Dairy cows \\
\hline
\end{tabular}

Original Research Article, Animal Science

*Corresponding author:

Oscar Balocchi

E-mail address:

obalocch@uach.cl

\begin{abstract}
A B S T R A C T
A deeper knowledge on the effects of herbage allowance (HA) on pasture characteristics and animal performance is necessary to improve efficiency of dairy production systems based on intensive grazing of pasture. The objectives of this study were to assess the effect of two levels of HA on pasture characteristics and milk performance during a three years period. Holstein Friesian dairy cows were selected from a dairy herd owned by the Universidad Austral de Chile and randomly assigned to one of two homogeneous groups. Each group was managed with different HA (high or low), measured at ground level, in a grazing system with half day strip. Grazing management variables (pre and postgrazing herbage mass, stocking rate, and proportion of pasture harvested), and animals variables (body weight, body condition score, milk production and composition), were measured and the results were averaged for each season of the year. Pre-grazing herbage mass did not differ between treatments averaging 2,313,2,261, 2,069 and 1,315 $\mathrm{kg} \mathrm{DM} \mathrm{ha}^{-1}$, for spring, summer, autumn and winter, respectively. Cows grazing at low HA had a lower post-grazing herbage mass (-146 $\left.\mathrm{kg} \mathrm{DM} \mathrm{ha}^{-1}\right)$, especially in summer and spring. Consequently the proportion of pasture harvested was greater at low HA (+5.5\%). Milk production per cow was not significantly reduced when a low level of HA was offered. However, due to higher stocking rate, milk production per hectare increased in low HA by $26 \%$ as average of the three-year period. Results suggest that the restriction on herbage allowance might be a useful grazing management tool to improve the profitability of dairy systems based on intensive grazing of pasture.
\end{abstract}

\section{RESUMEN}

Un mayor conocimiento de los efectos de la oferta de pradera (OP) sobre las características de la pradera y productividad animal son necesarios para mejorar la eficiencia de los sistemas de producción de leche basados en pastoreo. Los objetivos de este estudio fueron evaluar el efecto de dos niveles de OP sobre variables de la pradera y productividad de vacas lecheras, durante un período de tres años. Se utilizaron vacas lecheras Holstein Friesian pertenecientes al rebaño de la Universidad Austral de Chile. Las vacas se separaron en dos grupos homogéneos. Cada grupo fue manejado en una oferta de pradera diferente (alta o baja), medida a ras de suelo, en un sistema de pastoreo con franjas de medio día. Las variables de manejo del pastoreo (fitomasa pre y post-pastoreo, carga animal y proporción de cosecha de la pradera) y las variables de los animales (peso vivo, condición corporal, producción y composición de la leche), fueron medidos y los resultados promediados para cada estación del año. La fitomasa pre-pastoreo no difirió entre los tratamientos promediando 2.313, 2.261, 2.069 y 1.315 kg MS ha-1 en primavera, verano, otoño e invierno, respectivamente. Las vacas que pastorearon una baja OP dejaron una fitomasa postpastoreo inferior (-146 $\left.\mathrm{kg} \mathrm{ha}^{-1}\right)$, especialmente en verano y primavera. Consecuentemente la proporción de pradera cosechada fue mayor cuando las vacas recibieron una baja OP $(+5,5 \%)$. La producción de leche por vaca no se redujo significativamente cuando se ofreció un menor nivel oferta de pradera. Sin embargo, debido al aumento en la carga animal, la producción de leche por hectárea al año, promedio de los tres años evaluados, aumentó en $26 \%$ en el tratamiento de baja OP. Los resultados sugieren que la restricción en la oferta de pradera puede ser una herramienta útil de manejo del pastoreo para mejorar la rentabilidad de los sistemas de producción de leche basados en pastoreo.

Palabras clave: Manejo del pastoreo, eficiencia de pastoreo, carga animal, vacas lecheras.

\section{INTRODUCCIÓN}

Los sistemas de producción de leche en base a pastoreo son una alternativa competitiva de producción en zonas de clima templado en la medida que la pradera presente un buen nivel de calidad y rendimiento de materia seca a lo largo del año, con una eficiente conversión de materia seca de pradera en leche. La 
competitividad de estos sistemas productivos depende de un eficiente uso de la pradera como principal recurso alimenticio a través de un adecuado manejo del pastoreo.

La oferta de pradera ha sido definida como la cantidad diaria de fitomasa ofrecida por animal en pastoreo (Combellas y Hodgson, 1979). Es estimada a nivel del suelo o a una altura de 4 o $5 \mathrm{~cm}$ suponiendo que el material por debajo de esta altura no se encuentra disponible para el pastoreo animal (Dillon, 2006). La oferta de pradera se calcula en función de la fitomasa presente en la pradera previo al ingreso de los animales al pastoreo y se controla mediante la asignación diaria de superficie por grupo de animales.

La oferta de pradera determina la cantidad de animales por unidad de superficie (carga animal instantánea) y permite controlar la proporción de la fitomasa y de la altura inicial que serán removidas durante el pastoreo, así como la intensidad del mismo. En consecuencia, resulta un factor determinante sobre el consumo voluntario y producción de leche de vacas en pastoreo (Peyraud et al., 1996; Dalley et al., 1999; Dillon, 2006; Kennedy et al., 2008; Curran et al., 2010).

Estudios previos han mostrado que se logran mayores niveles de consumo y producción de leche de vacas en pastoreo cuando se aumenta la OP (Peyraud et al., 1996; McEvoy et al., 2008; Bargo et al., 2002). Sin embargo, ofertas de pradera no restrictivas para el consumo también determinan un aumento en la cantidad de residuo post-pastoreo (Peyraud y Delaby, 2001; Lee et al., 2008) y una menor eficiencia de uso de la pradera ofrecida (proporción de forraje cosechado menor a 50\%; McGilloway y Mayne, 1996). En consecuencia, altas OP pueden generar un deterioro progresivo de la calidad nutritiva de la pradera para los siguientes pastoreos (Stakelum y Dillon, 2007). Por el contrario, se ha encontrado que bajas OP disminuyen el consumo y producción de leche de los animales en pastoreo mientras aumentan la eficiencia de utilización de las praderas y la producción de leche por hectárea (Baudracco et al., 2010; Peyraud y Delagarde, 2013).

Hasta el momento no existe consenso entre los investigadores del nivel de OP apropiado para compatibilizar los requerimientos de los animales y de las especies forrajeras que componen la pradera. Por otro lado, no existen estudios que evalúen la respuesta productiva de la pradera sometida durante un largo tiempo a diferentes niveles de OP. Frente a este escenario, resulta fundamental definir los niveles de OP que permitan un sistema productivo y sustentable en el tiempo.

El estudio tuvo por objetivo determinar el efecto de dos niveles de OP sobre las características de la pradera y variables de manejo del pastoreo, además de determinar el efecto sobre la producción de leche, peso vivo y condición corporal de los animales en pastoreo.

\section{MATERIAL Y MÉTODOS}

El estudio se realizó en la Estación Experimental Vista Alegre, de la Universidad Austral de Chile, situada 6 kilómetros al norte de la ciudad de Valdivia, Región de los Ríos, Chile $\left(39^{\circ} 47^{\prime} \mathrm{S}\right.$ y $73^{\circ} 14^{\prime}$ O) durante un periodo de 3 años (primavera de 2008 hasta la primavera de 2011). El sitio del ensayo corresponde a un suelo de la serie Valdivia (Typic Hapludand), de topografía ligeramente ondulada y sin problemas de drenaje.

El área experimental fue de 27 hectáreas de praderas polifíticas con predominio de ballica perenne (Lolium perenne L.), divididas en 22 potreros de aproximadamente 1,2 ha cada uno (13 de alta y 9 de baja oferta de pradera).

Se utilizaron vacas lecheras del genotipo HolsteinFriesian las que fueron aleatoriamente distribuidas en dos grupos de pastoreo según los días de lactancia, producción de leche, peso vivo y condición corporal de los animales al inicio de cada período experimental.

Los grupos de vacas fueron manejados con un sistema de pastoreo en franjas de medio día, de modo que accedieron a una nueva pradera después de cada ordeña. La superficie de cada franja de pastoreo fue determinada en función de la oferta de pradera asignada a cada grupo de pastoreo y a la fitomasa presente en la pradera previo al inicio del pastoreo. Las vacas fueron ordeñadas dos veces al día (06:00 horas y 14:00 horas) y suplementadas con concentrado al momento de la ordeña.

El Cuadro 1 detalla las cantidades diarias de ensilaje, concentrado y la oferta de pradera utilizados en cada estación durante los 3 años de estudio. La composición nutritiva de la pradera fue similar para las dos ofertas en gran parte de sus constituyentes nutricionales. El concentrado utilizado fue de tipo energético $\left(3,2 \mathrm{Mcal} \mathrm{kg}^{-1}\right.$ de EM) y bajo de PC (14\%), basado en granos de cereales similar a los normalmente utilizados en el sur de Chile. El ensilaje fue de pradera permanente dominada por ballica perenne y premarchito con una composición promedio de $29 \%$ de MS, $12 \%$ de PC y 2,5 $\mathrm{Mcal} \mathrm{kg}^{-1}$ de EM.

Los niveles de oferta de pradera utilizados en cada estación del año variaron en función de las tasas de crecimiento de la pradera. Las cantidades de oferta de praderas ofrecidas en los distintos años de evaluación fueron ajustadas en función de la cantidad de pradera disponible y basado en la experiencia de la temporada anterior, manteniéndose la proporción entre ambas ofertas de pradera durante todo el período en estudio. Los niveles de suplementación con ensilaje y concentrado se calcularon en función de los requerimientos de los animales en pastoreo y de la cantidad de oferta de pradera asignada en cada estación del año.

\section{Evaluaciones}

Fitomasa de pre-pastoreo y post-pastoreo (residuo). La fitomasa ( $\mathrm{kg} \mathrm{MS} \mathrm{ha}^{-1}$ ) presente en las praderas antes y 
Cuadro 1. Cantidades diarias de ensilaje (kg base MS), concentrado ( $\mathrm{kg}$ base tal como ofrecido) y la oferta de la pradera (kg MS vaca ${ }^{-1}$ día $\left.^{-1}\right)$.

Table 1. Daily silage quantity (kg DM base), concentrate (kg as offered) and daily pasture allowance ( $\mathrm{kg} \mathrm{DM} \mathrm{cow}{ }^{-1}$ day $\left.^{-1}\right)$

\begin{tabular}{cc}
\hline Estación del año & Primavera Verano Otoño Invierno \\
$\left(\mathrm{kg} \mathrm{MS} \mathrm{vaca}^{-1} \mathrm{dí}^{-1}\right)$
\end{tabular}

Año 1

(2008-2009)

$\begin{array}{lcccc}\text { Ensilaje } & 0 & 6 & 4 & 7 \\ \begin{array}{l}\text { Concentrado } \\ \text { Oferta pradera }\end{array} & 3 & 3 & 3 & 3 \\ \quad \text { Alta oferta } & 38 & 26 & 30 & 25 \\ \quad \text { Baja oferta } & 26 & 14 & 20 & 16\end{array}$

Año 2

(2009-2010)

Ensilaje

Concentrado

\section{0}

\section{3}

Oferta pradera

$\begin{array}{lllll}\text { Alta oferta } & 30 & 30 & 25 & 22 \\ \text { Baja oferta } & 20 & 20 & 17 & 15\end{array}$

Año 3

(2010-2011)

\begin{tabular}{lcccc} 
Ensilaje & 0 & 0 & 6 & 6 \\
Concentrado & 3 & 3 & 3 & 3 \\
$\begin{array}{l}\text { Oferta pradera } \\
\quad \text { Alta oferta }\end{array}$ & 30 & 30 & 25 & 25 \\
$\quad$ Baja oferta & 20 & 20 & 20 & 17 \\
\hline
\end{tabular}

después del pastoreo, fue estimada a ras de suelo en base a la altura comprimida medida por un plato medidor de praderas marca Jenquip $\AA$ (Rising plate meter, New Zealand) en cada episodio de pastoreo. Para la calibración del instrumento, se utilizaron ecuaciones estacionales predeterminadas del proyecto "Validación y difusión de mejores prácticas de pastoreo para el sur de Chile", desarrollado en estas mismas praderas (Teuber et al., 2007).

Consumo aparente por animal (kg MS vaca día-1 ${ }^{-1}$. Se estimó en base a la cantidad de forraje aparentemente consumida por el grupo de vacas en pastoreo (diferencia entre fitomasa de pre-pastoreo y de residuo, kg MS $\mathrm{ha}^{-1}$ ), a la superficie diaria de pastoreo y al número de animales por tratamiento.

Proporción de forraje cosechado (\%). Corresponde a la relación entre el consumo aparente por hectárea (diferen- cia entre fitomasa presente al inicio y término del pastoreo) y la cantidad de fitomasa de pradera pre-pastoreo.

Producción de leche. Fue medida tres veces por semana con medidores tipo "Waikato" en ambas ordeñas.

Composición de la leche. Cada dos semanas se colectaron muestras de leche por vaca y se midió su composición (grasa y proteína) la que fue analizada mediante espectroscopia de infrarrojos (Foss 4300 Milko-scan), en el laboratorio de Cooprinsem en Osorno.

Peso vivo y condición corporal de los animales. Cada 15 días después de la ordeña matinal, se registró el peso vivo de los animales, mediante una balanza mecánica para $1500 \mathrm{~kg}$ (con una sensibilidad de $1 \mathrm{~kg}$ ). En la misma oportunidad se estimó la condición corporal a través de la escala de 1 a 5 , donde 1 es extremadamente delgado y 5 obeso (Ferguson et al., 1994).

\section{Diseño experimental y tratamientos}

El estudio consideró dos tratamientos determinados por la OP (alta y baja), en un diseño completamente al azar, considerándose las vacas y los potreros como las repeticiones para las variables animales y de la pradera, respectivamente. Los resultados fueron sometidos a análisis de varianza (ANDEVA) previa comprobación de los supuestos de normalidad y homogeneidad de varianza, utilizando el programa estadístico SAS (versión 9.1.3, SAS Institute Inc., 2006). El modelo estadístico utilizado para el análisis de las variables de la pradera fue:

$\mathrm{Y}_{i j}=\mu+\mathrm{T}_{i}+\xi_{i j,}$ donde, $\mathrm{Y}_{i j k}=$ variable dependiente (fitomasa pre $\mathrm{y}$ post-pastoreo, consumo aparente por vaca, proporción de forraje utilizado), $\mu$ = intercepto, $\mathrm{T}_{i}=$ efecto del i-ésimo tratamiento de oferta de pradera $(i=1,2), \xi_{i j}=$ residual del modelo.

Para el análisis de las variables relativas a los animales el modelo estadístico utilizado fue:

$\mathrm{Y}_{i j k}=\mu+\mathrm{T}_{\mathrm{i}}+\mathrm{W}_{\mathrm{j}}+\xi_{\mathrm{ijk}}$. Donde, $\mathrm{Y}_{i j k}=$ variable dependiente (producción de leche, \% grasa, \% proteína, peso vivo, condición corporal), $\mu=$ intercepto, $\mathrm{T}_{i}=$ efecto del iésimo tratamiento de oferta de pradera $(\mathrm{i}=1,2), \mathrm{W}_{j}=$ efecto de la j-ésima semana, $\xi_{i j k l}=$ residual del modelo.

\section{RESULTADOS Y DISCUSIÓN}

Los valores de fitomasa ( $\mathrm{kg} \mathrm{MS} \mathrm{ha}^{-1}$ ) pre-pastoreo y post-pastoreo, la carga animal (vaca ha ${ }^{-1}$ ) y el porcentaje de pasto cosechado por pastoreo (\%) promediados por estación para los tratamientos de alta y baja OP durante los 3 años del ensayo, se presentan en el Cuadro 2. 


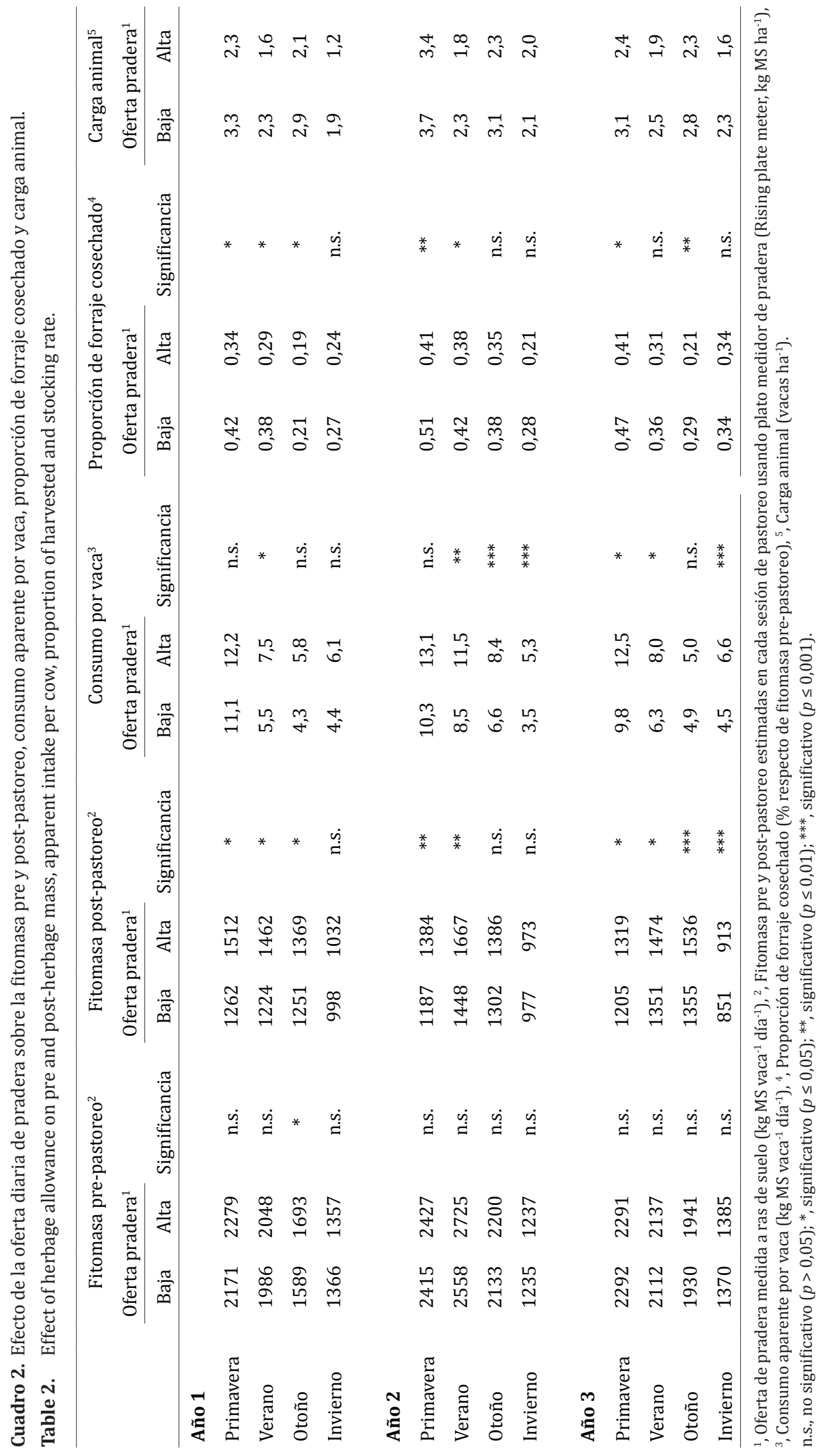


La fitomasa pre-pastoreo fue similar entre ambas tratamientos de OP durante todo el experimento, encontrándose una reducción significativa en los valores de fitomasa en las épocas de menor crecimiento de la pradera, especialmente en invierno. Estos valores corresponden a valores normales recomendados para el manejo del pastoreo en la zona sur de Chile a excepción de los meses de invierno (Teuber et al., 2007). Los valores menores de fitomasa pre-pastoreo encontrados en este estudio respecto a los recomendados durante los meses de invierno se debió a la baja tasa de crecimiento de las praderas durante este periodo. El grupo de vacas a las que se asignó una menor OP generaron un residuo post-pastoreo significativamente menor, particularmente durante los meses de mayor productividad de la pradera (primavera, verano y otoño). Consecuentemente, la proporción de pradera consumida por pastoreo fue mayor $(p \leq 0,05)$ en relación al tratamiento de alta OP. Las distintas OP dieron como resultado cargas animales disímiles con un promedio de los tres años de evaluación de 2,1 vs. 2,7 vacas por hectárea para alta y baja OP, respectivamente. Estas cargas disminuyeron durante los meses en los que la suplementación con ensilaje fue mayor (verano e invierno). En general, el grupo de baja OP mantuvo una carga animal de alrededor de un $30 \%$ superior en relación a la alta OP.

El efecto de la OP sobre la producción de la leche por animal y composición láctea se muestra en el Cuadro 3. Se observa que como promedio durante los tres años de evaluación, se produjo un leve aunque no significativo aumento en la producción de leche individual en respuesta al aumento en la OP $(+0,07 \mathrm{~L}$ por $\mathrm{kg}$ de aumento en la OP). La ausencia de diferencia en la producción y composición de leche encontrada en este estudio es consistente con los resultados publicados por Kennedy et al. (2008), evaluando ofertas, medidas a $4 \mathrm{~cm}$ de altura, de 13, 16 y $19 \mathrm{~kg}$ MS vaca ${ }^{-1}$ día $^{-1}$ en vacas a inicios y mediados de lactancia, quienes no encontraron efectos significativos de la oferta de pradera en la producción individual de leche. Sin embargo, incrementos significativos en la producción de leche por vaca como resultado del aumento en la oferta de pradera se han visto en estudios que evaluaron ofertas de pradera más contrastantes (Stockdale et al., 2000; Bargo et al., 2002; Pérez-Prieto et al., 2011).

Durante los meses de primavera fue cuando se observó la menor diferencia productiva $\left(0,3 \mathrm{~L} \mathrm{vaca}^{-1}\right.$ día $\left.^{-1}\right)$ entre ambas ofertas. Probablemente, una alta carga animal en primavera puede no necesariamente afectar la producción de leche por vaca, debido al aumento de la digestibilidad y densidad de la pradera y a una disminución de las áreas rechazadas en la misma (Backer et al., 1986).

La producción anual de leche por hectárea, aumentó al reducir la OP desde 16.339 a $20.624 \mathrm{~L}$, como promedio de los tres años (Figura 1). Este aumento se explica por la mayor carga animal que se produce al restringir la oferta diaria de pradera, sin un efecto muy relevante en la producción individual de leche de las vacas. La carga animal es considerada el principal impulsor de la producción de leche por hectárea y consecuentemente de la rentabilidad de los sistemas de pastoreo (Baudracco et al., 2010). En consecuencia, el incremento en la carga animal que se genera al restringir la $\mathrm{OP}$ aumentó la producción de leche por hectárea en un $26 \%$, lo cual representa un importante incremento de la rentabilidad del sistema lechero.

En la mayoría de las estaciones no se encontró un efecto significativo de la OP sobre el contenido de grasa y proteína de la leche. Sólo en invierno del primer año y en otoño del segundo año la concentración de grasa y proteína fue mayor en el tratamiento con alta oferta de pradera. Como promedio del periodo evaluado la grasa fue 0,1 unidad de porcentaje y la proteína 0,06 unidades de porcentaje superiores en el tratamiento con mayor oferta de pradera (Cuadro 3). La ausencia de un efecto significativo de la OP sobre el contenido de grasa y proteína en la leche fue previamente descrita por Burke et al. (2008)

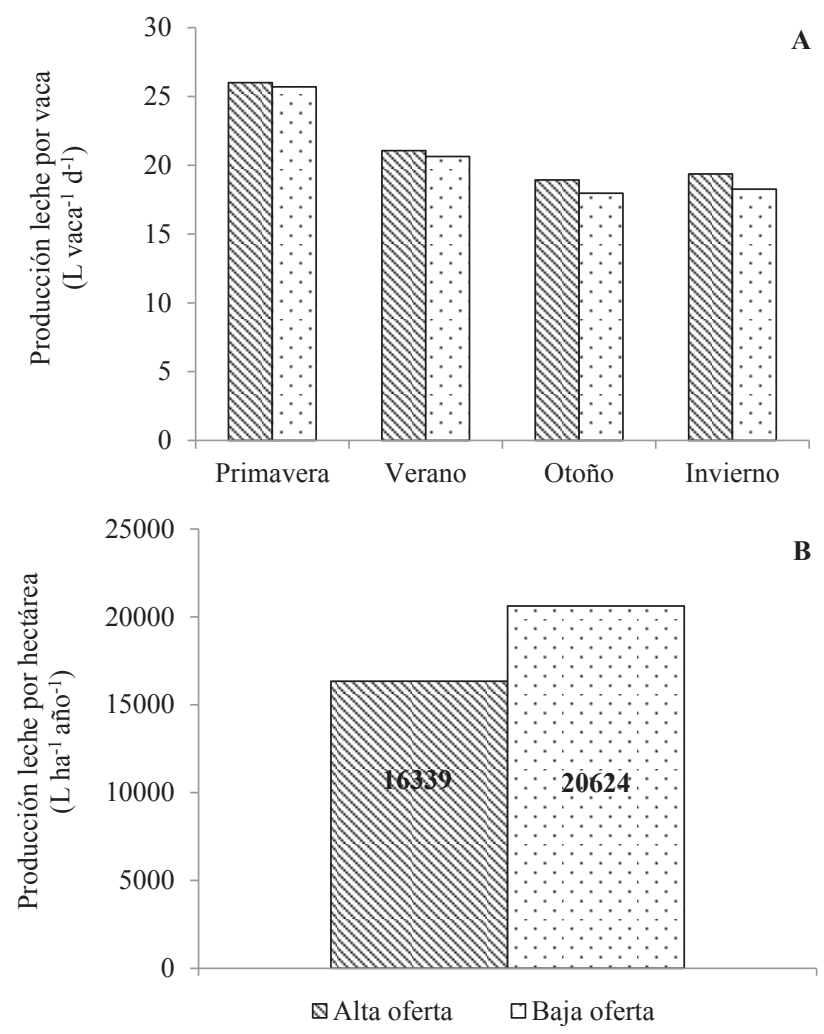

Figura 1. A) Producción de leche promedio estacional por vaca ( $\mathrm{L} \mathrm{vaca}^{-1}$ día $\left.^{-1}\right)$; B) Producción de leche por hectárea ( $\mathrm{L} \mathrm{ha}^{-1}$ período $^{-1}$ ). Valores promedio de los tres años de evaluación.

Figure 1. A) Average of seasonal milk production per cow $\left(\mathrm{L}\right.$ cow $^{-1}$ day $\left.^{-1}\right)$; B) Milk production per hectare ( $\mathrm{L} \mathrm{ha}^{-1}$ pe$\operatorname{riod}^{-1}$ ). Average values for three years of evaluation. 


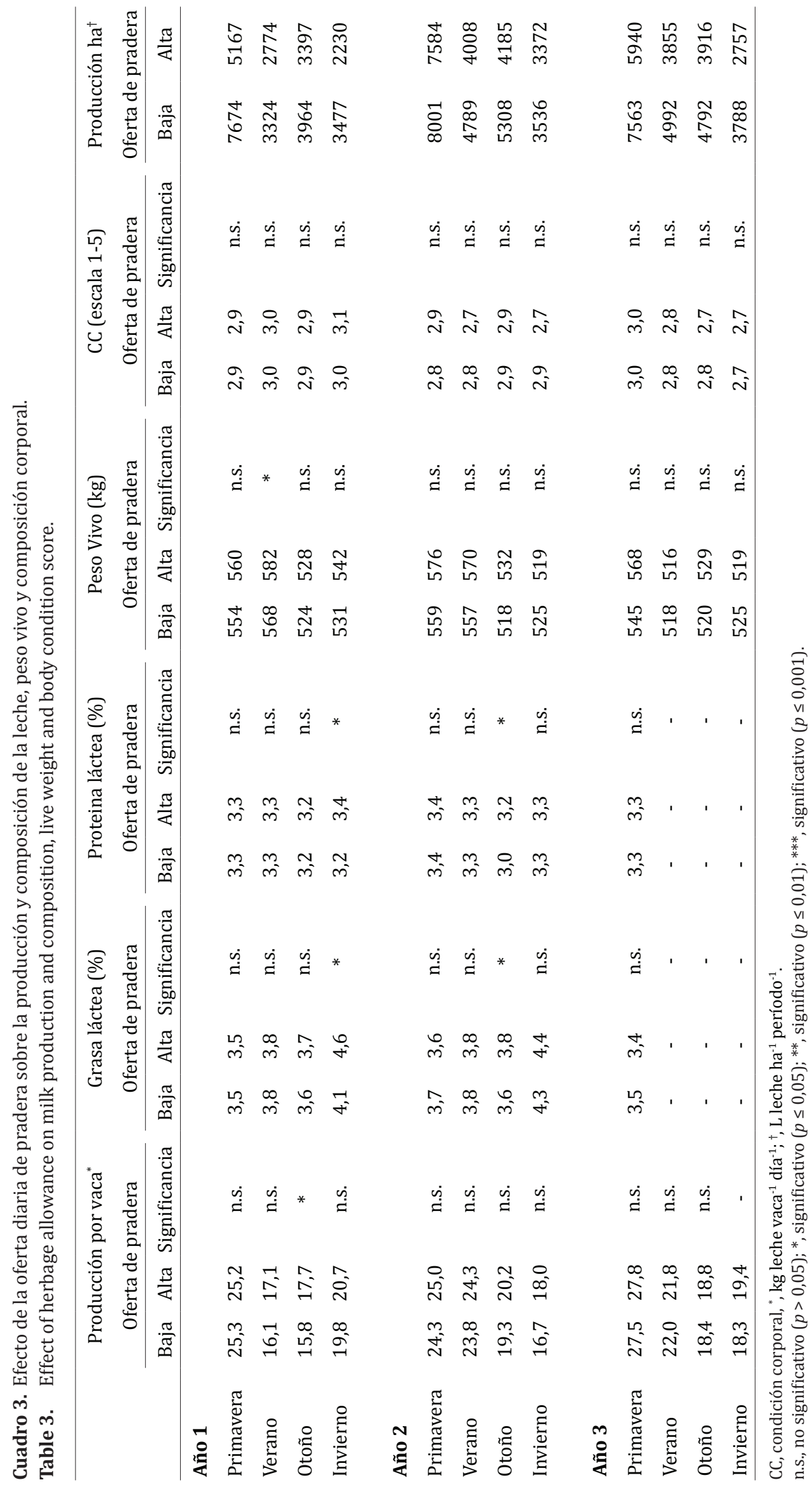


y Delaby et al. (2003) en vacas a pastoreo usando bajos niveles de suplementación energética (< 6 kg por vaca).

Los resultados del peso vivo y condición corporal promedio por estación se muestran en el Cuadro 3. No existieron diferencias significativas en el peso vivo de las vacas entre ambas ofertas de pradera, con excepción del verano del año 1, donde las vacas en alta OP tuvieron un peso promedio de $14 \mathrm{~kg}$ el peso superior que las vacas en baja OP. Posiblemente esto pueda deberse a que la menor oferta utilizada en ese período resultó restrictiva para el consumo animal. Sin embargo, la CC no fue afectada por la oferta de pradera durante los tres años de estudio, lo cual reflejaría que la condición corporal es menos sensible a los cambios en la oferta de pradera.

\section{CONCLUSIONES}

La restricción de la oferta diaria de pradera aumentó la eficiencia de uso de la pradera y la producción de leche por hectárea, sin que la producción individual, la composición de la leche, el peso vivo y la condición corporal de los animales en pastoreo fuesen significativamente deprimidos. En consecuencia, es posible aumentar la productividad por unidad de superficie en sistemas de producción de leche a pastoreo a partir de la restricción en la cantidad de pradera ofrecida por animal.

\section{AGRADECIMIENTOS}

Este trabajo fue parte del proyecto M1P2 "Identificación y monitoreo de sistemas de producción de leche competitivos por macrozonas homogéneas en Chile" del Consorcio Lechero, cuyo responsable fue el Dr. Francisco Lanuza de INIA Remehue, a quien se agradece su valiosa contribución.

\section{REFERENCIAS}

BAKER, A., MARIE, C., LEAVER, J. 1986. Effect of stocking rate in early season on dairy cow performance and sward characteristics. Grass and Forage Science 41(4): 333-340.

BARGO, F., MULLER, L.D., DELAHOY, J.E., CASSIDY, T.W. 2002. Milk Response to Concentrate Supplementation of High Producing Dairy Cows Grazing at Two Pasture Allowances. Journal of Dairy Science 85: 1777-1792.

BAUDRACCO, J., LÓPEZ-VILLALOBOS, N., HOLMES, C. W., MACDONALD, K. A. 2010. Effects of stocking rate, supplementation, genotype and their interactions on grazing dairy systems: A review. New Zealand Journal of Agricultural Research 53: 109-133.

BURKE, F., O'DONOVAN, M.A., MURPHY, J.J., O'MARA, F.P., MULLIGAN, F.J. 2008. Effect of pasture allowance and supplementation with maize silage and concentrates differing in crude protein concentration on milk production and nitrogen excretion by dairy cows. Livestock Science 114: 325-335.

COMBELLAS, J., HODGSON, J. 1979. Herbage intake and milk production by grazing dairy cows. The effects of variation in herbage mass and daily herbage allowance in a short-term trial. Grass Forage Science 34: 209-214.

CURRAN, J., DELABY, L., KENNEDY, E., MURPHY, J.P., BOLAND, T.M. Y O'DONOVAN, M. 2010. Sward characteristics, grass dry matter intake and milk production performance are affected by pre-grazing herbage mass and pasture allowance. Livestock Science 127: 144-154.

DALLEY, D.E., ROCHE, J.R., GRAINGER, C., MOATE, P.J. 1999. Dry matter intake, nutrient selection and milk production of dairy cows grazing rainfed perennial pastures at different herbage allowances in spring. Australian Journal of Experimental Agriculture 39: 923-931.

DELABY, L., PEYRAUD, J.L., DELAGARDE, R. 2003. Faut-il complémenter les vaches laitières au pâturage? INRA. Productions Animales 16(3): 183-195.

DILLON, P. 2006. Achieving high dry-matter intake from pasture with grazing dairy cows. Fresh Herbage for Dairy Cattle. Elgersma, A., Dijkstra, J., Tamminga, S. (Eds.), Fresh Herbage for Dairy Cattle, Springer, Netherlands, pp. 1-26.

FERGUSON, J.D., GALLIGAN D.T., THOMSEN, N. 1994. Principal descriptors of body condition score in Holstein cows. Journal of Dairy Science 77: 2695-2703.

KENNEDY, E., O’DONOVAN, M., DELABY, L., O'MARA, F.P. 2008. Effect of herbage allowance and concentrate supplementation on dry matter intake, milk production and energy balance of early lactating dairy cows. Livestock Science. 117: 275-286.

LEE, J.M., DONAGHY, D.J., ROCHE, J. R. 2008. Short Communication: Effect of Postgrazing Residual Pasture Height on Milk Production. Journal of Dairy Science 91: 4307-4311.

McEVOY, M., KENNEDY, E., MURPHY, J.P., BOLAND, T.M., DELABY, L., O'DONOVAN, M. 2008. The effect of herbage allowance and concentrate supplementation on milk production performance and dry matter intake of spring-calving dairy cows in early lactation. Journal of Dairy Science 91: 1259-1269.

McGILLOWAY, D.A., MAYNE, C.S. 1996. The importance of grass availability for the high genetic merit dairy cows. In: Garnsworthy, P.C., Wiseman, J. (Eds.), Recent Advances in Animal Nutrition. Nottingham University Press, Nottingham (UK), pp. 135-169.

PÉREZ-PRIETO, L.A., PEYRAUD, J.L., DELAGARDE, R. 2011. Pasture intake, milk production and grazing behavior of dairy cows grazing low-mass pastures at three daily allowances in winter. Livestock Science 137: 151-160. 
PEYRAUD, J.L., COMERON, E.A., WADE, M.H., LEMAIRE, G. 1996. The effect of daily herbage allowance, herbage mass and animal factors upon herbage intake by grazing dairy cows. Annales de Zootechnie 45: 201-217.

PEYRAUD, J.L., DELABY, L. 2001. Ideal concentrate feeds for grazing dairy cows responses to supplementation in interaction with grazing management and grass quality. In: Garnsworthy, P.C., Wiseman, J. (Eds.), Recent Advances in Animal Nutrition. Nottingham University Press, Nottingham (UK), pp. 203-220.

PEYRAUD, J.L., DELAGARDE, R. 2013. Managing variations in dairy cow nutrient supply under grazing. Animal (Suppl. s1): 57-67.
STAKELUM, G., DILLON, R. 2007. The effect of grazing pressure on rotationally grazed pastures in spring/early summer on the performance of dairy cows in the summer/ autumn period. Irish Journal of Agricultural and Food Research 46: 29-46.

STOCKDALE, C. R. 2000. Levels of pasture substitution when concentrates are fed to grazing dairy cows in northern Victoria. Australian Journal of Experimental Agriculture 40: 913-921.

TEUBER, N., O. BALOCCHI, O., PARGA, J. 2007. Manejo del pastoreo. Fundación para la Innovación Agraria, Instituto de Investigaciones Agropecuarias, Universidad de la Frontera, Universidad Austral de Chile, Cooprinsem, ANASAC, Chile. 